\title{
Macro-TSH: A Diagnostic Challenge
}

\author{
Camilla Bøgelund Larsen ${ }^{a, b}$ Eva Rabing Brix Petersen ${ }^{c}$ Martin Overgaard ${ }^{b, d}$ \\ Steen Joop Bonnema ${ }^{a, b}$ \\ ${ }^{a}$ Department of Endocrinology, Odense University Hospital, Odense, Denmark; ${ }^{b}$ Department of Clinical Research, \\ University of Southern Denmark, Odense, Denmark; ' Department of Clinical Biochemistry and Immunology, \\ Hospital of Southern Jutland, Aabenraa, Denmark; ${ }^{d}$ Department of Clinical Biochemistry and Pharmacology, \\ Odense University Hospital, Odense, Denmark
}

\section{What Is Known about This Topic?}

- Macro-TSH is a rare condition, which is caused by binding of TSH to other plasma proteins, most often immunoglobulins. This results in falsely elevated TSH measurement.

- The biochemical profile mimics subclinical hypothyroidism and may lead to inappropriate LT4 treatment.

- No immunoassay can reveal the presence of macro-TSH. Gel filtration chromatography is the stateof-the-art method for detection of macro-TSH.

\section{What Does This Case Report Add?}

- This report highlights the importance of screening for macro-TSH, in addition to other analytical problems, in patients with a markedly and isolated elevated plasma TSH, particularly if other signs of thyroid dysfunction are absent.

\section{Keywords}

Macro-TSH · Analytic errors · Gel filtration chromatography land). The patient's complaints were unspecific, and he appeared clinically euthyroid. The plasma levels of free T4 and free T3 were within the normal range, thyroid autoantibodies were negative, and thyroid ultrasonography was normal. During a short trial of thyroid hormone substitution, the level of TSH decreased to near-normal levels, but hyperthyroid symptoms emerged. TSH analysed by a different immunoassay (Architect; Abbott, Chicago, IL, USA) yielded similar results. In addition, serial dilutions were performed showing linearity, without detection of heterophilic antibody interference. Gel filtration chromatography confirmed the presence of macro-TSH. Conclusion: The patient har- 
boured macro-TSH, which is a rare condition. The complex binding of TSH to other plasma proteins, most often immunoglobulins, results in elevated plasma TSH. However, the biologically active fraction of TSH is normal, reflected by clinical and biochemical euthyroidism.

(C) 2020 European Thyroid Association Published by S. Karger AG, Basel

\section{Introduction}

Despite methodological improvements, immunoassays used for evaluation of the thyroid function are still exposed to different types of interferences. Macro-thyrotropin (macro-TSH) is caused by a large amount of monomeric TSH complexed with anti-TSH antibodies mostly immunoglobulin $\mathrm{G}[1,2]$. Macro-hormones, including macro-TSH, are considered biologically inactive, and only a small fraction of the total amount of TSH in plasma remains free [3].

Although being bound into such complexes, TSH is still detected by the current assays used for TSH measurement, causing elevated results. Thus, none of the commercially available immunoassays employed for TSH testing can discriminate macro-TSH from the bioactive freely circulating TSH. In patients harbouring macro$\mathrm{TSH}$, the falsely elevated TSH may lead to clinical mismanagement. Macro-TSH is a rare condition, with a reported prevalence ranging from 0.6 to $1.6 \%$ [4-6]. Here, we present a case with isolated TSH elevation, illustrating the challenges of diagnosing macro-TSH.

\section{Case Presentation}

A 29-year-old male was referred from a local hospital with persistently elevated TSH (148 mIU/L) measured by a sandwich electrochemiluminescence immunoassay (ECLIA) on Cobas 8000 (Roche, Basel, Switzerland). The patient's complaints were unspecific of nature, like tiredness, headache, dizziness, and discomfort in the chest. Plasma levels of free T4 and free T3 were within the normal range, and thyroid autoantibodies were negative. The physical examination was without abnormal findings, and he appeared clinically euthyroid. Thyroid ultrasonography was normal regarding size, texture, and echogenicity. In addition, MRI of the pituitary gland and an ACTH-stimulation test were normal. The symptoms and the increased TSH had prompted a trial of levothyroxine (LT4) replacement therapy on the suspicion of thyroid failure. Up to $150 \mu \mathrm{g}$ LT4 per day was given. Hereby, plasma TSH decreased to near-normal levels within a few weeks, but hyperthyroid symptoms emerged in parallel with the thyroid hormones rising to supranormal levels (Fig. 1). Therefore, the treatment was withdrawn.
Supplementary Laboratory Tests

Measurement of TSH by use of a different immunoassay (Architect; Abbott, Chicago, IL, USA) yielded a similar high TSH level ( $>100 \mathrm{mIU} / \mathrm{L})$ as did the assay from Roche. Serial dilution of the patient's plasma was analysed using both immunoassays to rule out possible interference from human anti-mouse antibodies targeting TSH assay antibodies. For both assays, linear and almost identical dilution patterns were found (Fig. 2a), making interference from heterophilic antibody highly unlikely. Further analyses included search for a mutation in the TSH-receptor-gene and the TSH-beta-gene, also with negative results [7]. Plasma TSH in both parents was within the reference range.

Thyroid failure was considered unlikely, based on the current results, the very high TSH level contrasting the normal levels of free T3 and free T4, as well as the appearance of the patient. The possibility of a complex formation (i.e., macro-TSH) of TSH and an unknown component in the plasma was considered. Therefore, gel filtration chromatography (GFC) was performed on plasma samples from the patient, a control person with slightly elevated $\mathrm{TSH}$, and a pool of healthy controls, respectively (Fig. 2b). A total of $100 \mu \mathrm{L}$ plasma was fractioned on a Superdex S-75 column (PBS, pH 7.4; 1 mM EDTA) via ÄKTA FPLC. Fractions of $500 \mu \mathrm{L}$ were collected, and TSH was analysed by Cobas 8000 e801 (Roche, Basel, Switzerland). The TSH peak of the control person eluted at 24 $\mathrm{kDa}$, corresponding to free TSH (theoretically $28 \mathrm{kDa}$ ), while the patient's TSH peak eluted at $154 \mathrm{kDa}$, compatible with a TSH-IgG complex (theoretically $178 \mathrm{kDa}$ ). This finding confirmed that the highly elevated plasma TSH was caused by the presence of macroTSH. Thus, the patient had no thyroid disorder and his symptoms were non-thyroid related.

\section{Discussion}

Characteristics of patients with macro-TSH include elevated TSH, normal thyroid hormone levels, and no clinical symptoms of thyroid dysfunction. Plasma TSH is often markedly increased, like in our patient, but cases with only slightly elevated TSH have been published [5]. Biochemically, this mimics subclinical hypothyroidism and may lead to inappropriate LT4 therapy [2]. However, a decrease in plasma TSH in response to LT4 therapy cannot exclude the presence of macro-TSH $[5,8]$. As demonstrated in our case, plasma TSH decreased significantly within a few weeks during LT4 replacement therapy, reflecting a suppression of TSH secretion of the pituitary gland. This observation seems to indicate that TSH is bound rather weakly to the immunoglobulins and is in a state of equilibrium with the free TSH in the circulation. If the complex binding was irreversible, the decline in TSH would probably have been more prolonged, considering the long half-life of immunoglobulins. To a large extent, macro-TSH mimics the situation seen with the thyroid hormones, where only a minor fraction is freely circulating due to protein binding. 


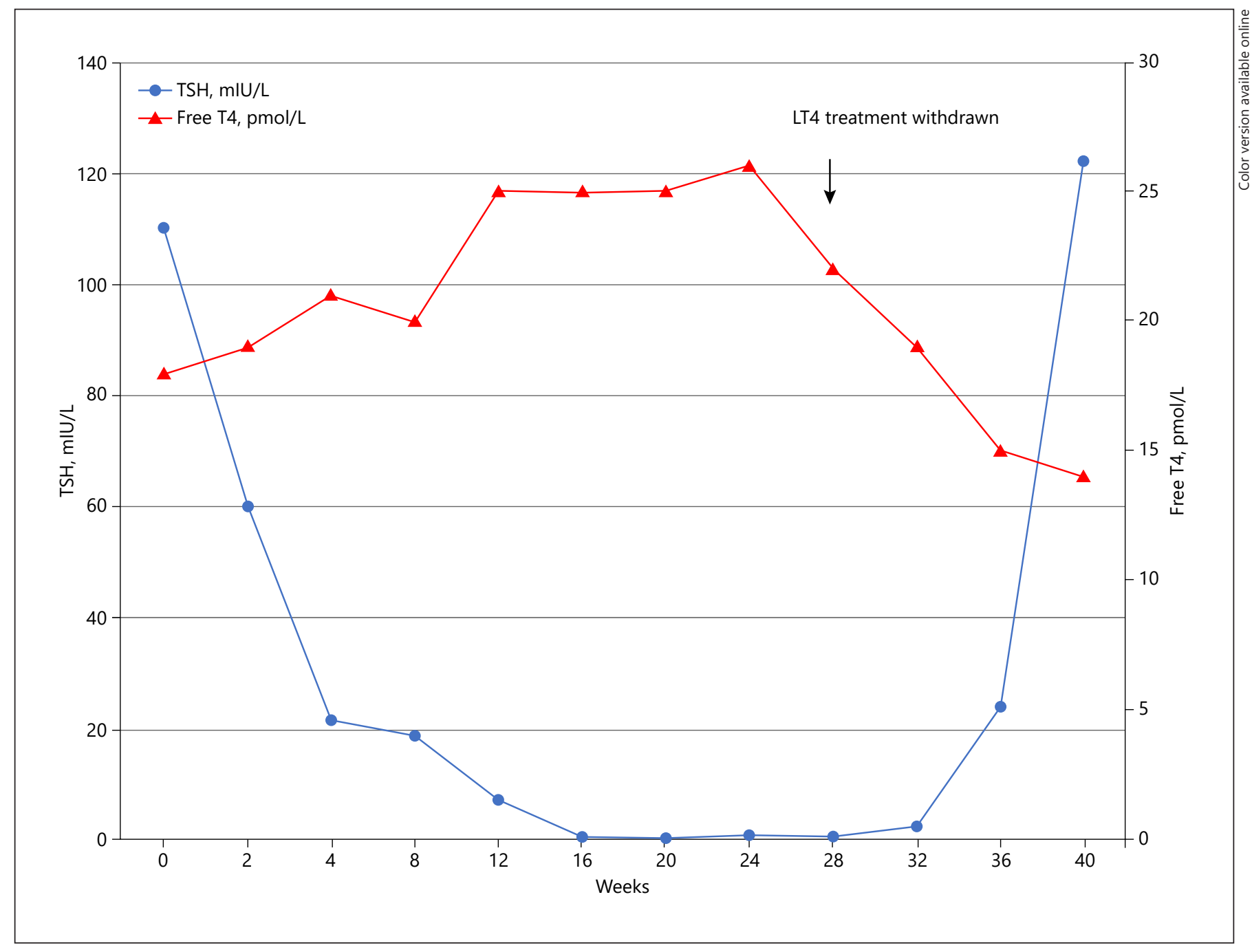

Fig. 1. The response to LT4 replacement therapy in the patient with macro-TSH. Initially, plasma TSH was high but decreased during LT4 replacement therapy, in parallel with a rise in plasma free T4. Reference interval of TSH: 0.3-4.0 mIU/L; free T4: 12-21 pmol/L. LT4 was initiated at week $=0$ and withdrawn at week $=28$. LT4, levothyroxine.

No routine TSH immunoassay can disclose the presence of macro-TSH. For this purpose, the gold standard method is GFC, which can separate the various TSH fractions according to the molecular weight. There is no clear answer when to screen for macro-TSH. This condition may be more common than hitherto reported. Mills et al. [6] suggested that macro-TSH should be suspected in case of TSH above $10 \mathrm{mIU} / \mathrm{L}$, while Hattori et al. [4] recommended screening for macro-TSH, especially in women of childbearing age in whom LT4 therapy for subclinical hypothyroidism is considered. Since macro-TSH is present in less than $1 \%$ in most populations, such an ap- proach is controversial and will significantly increase the costs of thyroid function tests.

For screening of macroprolactinemia, polyethylene glycol (PEG)-mediated precipitation has been widely adopted by clinical laboratories [9]. This method is based on either the post-PEG recovery level of monomeric prolactin or the use of post-PEG monomeric prolactin reference intervals. Dilution studies and measurement of TSH after addition of PEG might be helpful in identifying assay interference, without being definitive regarding the cause of such interference. Considering lower costs and higher accessibility than with GFC, such an approach may be a 
Fig. 2. a Serial dilution analyses were made with two different assays (Cobas and Architect), both showing similar and normal linear TSH dilution response. The upper limit of measurement in both assays is 100 $\mathrm{mIU} / \mathrm{L}$, undiluted measurements are thus not shown. b Analysis of TSH monomer and macro species in plasma. Plasma (0.1 $\mathrm{mL}$ ) from the patient (TSH case), a control person with slightly elevated TSH, and a pool of healthy controls, respectively, was subjected to GFC. TSH immunoreactivity was analysed in the resulting fractions using the Cobas method. The expected (interpolated) molecular weights $(\mathrm{kDa})$ of free TSH and TSH bound to IgG are indicated by arrows. GFC, gel filtration chromatography; IgG, immunoglobulin G.

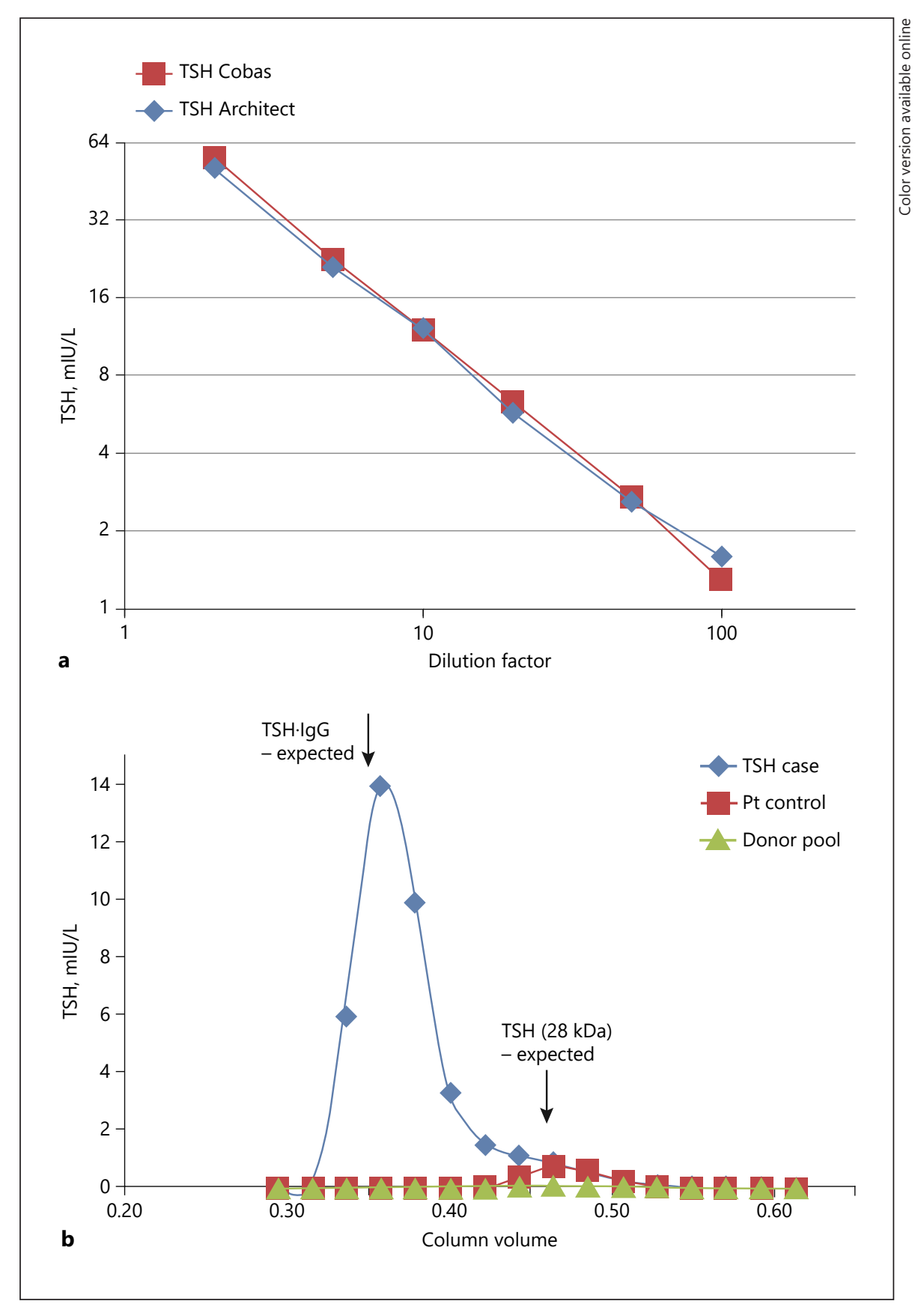

valid alternative for detection of macro-TSH, although results must be confirmed by performing GFC [2].

The aetiology behind the development of macro-TSH is unknown. Likewise, little is known whether macroTSH may persist lifelong or if it is transient of nature. Apart from jeopardizing the diagnostic process, the presence of macro-TSH is probably of no harm to the patient.
Nevertheless, it may be considered to follow up the patients regularly, in order to gain more knowledge about this condition $[1,10]$.

In conclusion, macro-TSH, in addition to other analytic interferences, should always be considered in patients with a markedly and isolated elevated plasma TSH, particularly if other signs of thyroid dysfunction are absent. 


\section{Acknowledgements}

We want to thank consultant Sevinch Rustamova, MD, who has been involved in the care taking of the patient and Niels Strømvig Larsen for excellent technical assistance.

\section{Statement of Ethics}

The research was conducted ethically in accordance with the World Medical Association Declaration of Helsinki. The patient gave consent to publication of the case.

\section{Disclosure Statement}

The authors have no conflicts of interest to declare.

\section{Funding Sources}

There were no funding sources.

\section{Author Contributions}

All authors participated in drafting the article or revising it critically for important intellectual content and gave their final approval of the version to be submitted.

\section{References}

1 Hattori N, Ishihara T, Matsuoka N, Saito T, Shimatsu A. Anti-Thyrotropin Autoantibodies in Patients with Macro-Thyrotropin and Long-Term Changes in Macro-Thyrotropin and Serum Thyrotropin Levels. Thyroid. 2017 Feb;27(2):138-46.

2 Favresse J, Burlacu MC, Maiter D, Gruson D. Interferences With Thyroid Function Immunoassays: Clinical Implications and Detection Algorithm. Endocr Rev. 2018 Oct;39(5):83050.

3 Hattori N, Aisaka K, Chihara K, Shimatsu A. Current Thyrotropin Immunoassays Recognize Macro-Thyrotropin Leading to Hyperthyrotropinemia in Females of Reproductive Age. Thyroid. 2018 Oct;28(10):1252-60.
4 Hattori N, Ishihara T, Shimatsu A. Variability in the detection of macro TSH in different immunoassay systems. Eur J Endocrinol. 2016 Jan;174(1):9-15.

5 Hattori N, Ishihara T, Yamagami K, Shimatsu A. Macro TSH in patients with subclinical hypothyroidism. Clin Endocrinol (Oxf). 2015 Dec;83(6):923-30.

6 Mills F, Jeffery J, Mackenzie P, Cranfield A, Ayling RM. An immunoglobulin G complexed form of thyroid-stimulating hormone (macro thyroid-stimulating hormone) is a cause of elevated serum thyroid-stimulating hormone concentration. Ann Clin Biochem. 2013 Sep;50(Pt 5):416-20.

7 Stephenson A, Lau L, Eszlinger M, Paschke R. The Thyrotropin Receptor Mutation Database Update. Thyroid. 2020 Jun;30(6):931-5.
8 Verhoye E, Van den Bruel A, Delanghe JR, Debruyne E, Langlois MR. Spuriously high thyrotropin values due to anti-thyrotropin antibodies in adult patients. Clin Chem Lab Med. 2009;47(5):604-6.

9 Overgaard M, Pedersen SM. Serum prolactin revisited: parametric reference intervals and cross platform evaluation of polyethylene glycol precipitation-based methods for discrimination between hyperprolactinemia and macroprolactinemia. Clin Chem Lab Med. 2017 Oct;55(11):1744-53.

10 Loh TP, Kao SL, Halsall DJ, Toh SA, Chan E, Ho SC, et al. Macro-thyrotropin: a case report and review of literature. J Clin Endocrinol Metab. 2012 Jun;97(6):1823-8. 